\title{
INHOMOGENEITIES IN BEAMS EXTRACTED FROM ECR ION SOURCES*
}

\author{
J. W. Stetson", NSCL/MSU, East Lansing, MI 48824, USA \\ P. Spädtke^, Gesellschaft für Schwerionenforschung, GSI Darmstadt, Germany
}

\section{Abstract}

An examination of heavy ion beam profiles using viewing targets and CCD cameras at both the NSCL and GSI shows highly structured patterns. These structures generally have a 3-fold symmetry reflecting the highlymagnetized nature of the ion formation within the plasma chamber. A program of experiment and modeling with KOBRA3-INP [1], TRANSPORT [2], GICOSY [3] is continuing. Results of this program to date are discussed.

\section{INTRODUCTION}

The National Superconducting Cyclotron Laboratory (NSCL) consists of two cyclotrons in series [4] which accelerate beams provided by one of two ECR Ion Sources. The primary source is ARTEMIS-A (Advanced Room Temperature Ion Source), which is a modification of the Berkeley AECR source and runs at $14 \mathrm{GHz}$ using permanent sextupole magnets, radial ports and room temperature solenoids. A $6.4 \mathrm{GHz}$ superconducting ion source serves primarily to produce the lighter, gas beams and as a backup to ARTEMIS. The experimental program requires ever-higher beam intensities to study the properties of exotic nuclei. However it was soon noted that increasing the source output did not proportionately increase beam output from the accelerators. It was also noted that images of the beam in the injection line using coated plates and video cameras displayed some surprising features that are difficult to discern using the more typical $(\mathrm{x}, \mathrm{y})$ wire scanners. As a result, considerable effort has been made to understand transport of beam from the ECR ion sources to the first cyclotron (K500). A drawing of the first half of the injection line is given in Figure 1.

\section{EXPERIENCE}

Beam simulations using TRANSPORT, which worked very well in the NSCL high-energy lines, worked very poorly when applied to the injection line. When handtuning, it was generally not possible to achieve even simple conditions such as a line focus using a quadrupole, where instead of collapsing to a line, the beam spot seen on the viewer would seem to rotate in a 3-d space. Even when severely restricted by apertures, odd behavior was noted and it was impossible to illuminate a spot evenly. Often, a star- shape was seen that was very similar to "burn" marks that are normally found on the inside ECRIS plasma chambers. This suggested that the failure of the optics code to predict beam envelopes was not a

*Supported under National Science Foundation Grant PHY-0110253

and EURONS

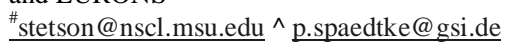

result of poor lenses, but a result of assuming a "wellassumption of a round, uncorrelated beam emission from the plasma zero-potential boundary, the so-called meniscus, while adequate for proton and electron sources, was no longer adequate.

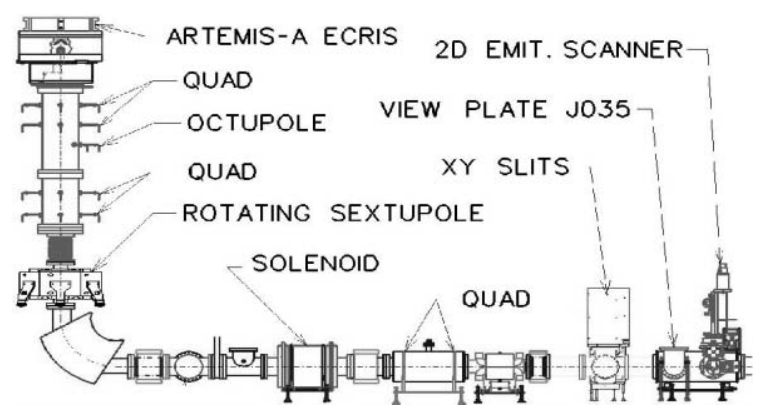

Figure 1: Injection beam line layout. The distance from ARTEMIS-A to the analysis magnet is about $2.2 \mathrm{~m}$.

\section{GSI Beam Tests}

The viewer plates at the NSCL are normally installed only at locations after the analysis magnet. It was desired to observe the beam properties directly outside of the source without intervening optical elements that impede understanding. The availability of the CAPRICE ECRIS mounted on an off-line diagnostic test stand along with the development of $\mathrm{a} \mathrm{BaF}_{2}$ scintillating material that could withstand high beam intensities for a reasonable length of time [5] allowed these observations to be done at GSI in July 2006.

The resulting images show that the beam is highly structured upon extraction from the ion source, as shown in Figure 2 (left). The triangular shape is pronounced (suggesting a strong $2^{\text {nd }}$ order aberration) and the beam is hollow. The detailed form can vary greatly as source parameters are changed (for example, the images rotate as the solenoid mirror field is varied), but the ring shape is generally retained unless the source is so mistuned that most beam intensity is lost.

Additionally, passing the beam though a solenoid results in a unique signature: the ring can be focused to what seems to be a decent spot, but stronger focusing turns that ring into a "star" as in Figure 2 (right). An uncorrelated ring would be expected to pass from a ring to another, inverted, ring as the solenoid strength is raised. This is certainly not the case here, which indicates that the extracted particles retain a "memory" of their formation in magnetic fields. 

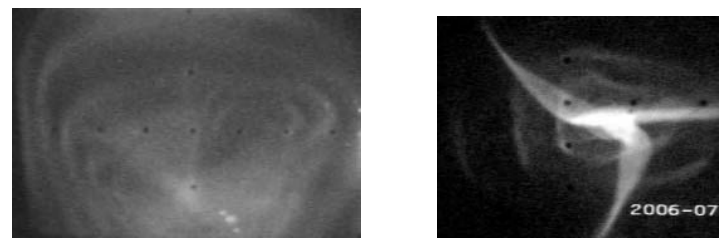

Figure 2: Viewer plate images of a ${ }^{40} \mathrm{Ar}$ beam from the CAPRICE source at GSI. The left viewer image is located $50 \mathrm{~cm}$ downstream of the extraction aperture. On the right is an image $2 \mathrm{~m}$ further downstream after focusing by a solenoid. The 2 rings and star are 3 charge states being focused differently by the solenoid.

\section{NSCL Beam Images}

A similar ring structure is seen on a viewer temporarily installed before the bending magnet and downstream of the focusing solenoid on ARTEMIS-B, as shown in Figure 3 .

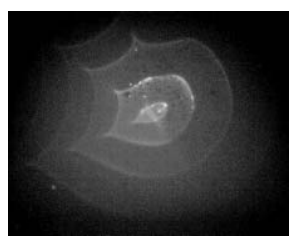

Figure 3: ${ }^{40} \mathrm{Ar}$ rings from Artemis-B on the test stand. The obvious distortion is caused by current leads feeding into the end of the solenoid.

Once it was realized that object of the transport optics was ring-like rather than disc-like, it became easy to find images of that shape (Figure 4).

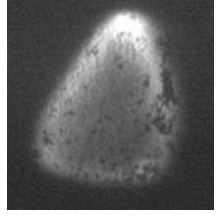

Figure 4: ${ }^{48} \mathrm{Ca}^{8+}$ rings from Artemis-A propagating through various optical elements down the beam line at $\mathrm{z}$ $=10 \mathrm{~m}, 13 \mathrm{~m}$, and $16 \mathrm{~m}$, respectively.

Attempting to limit emittance by use of apertures meets with only limited success, because the beam seems to have a fractal nature (Figure 5).
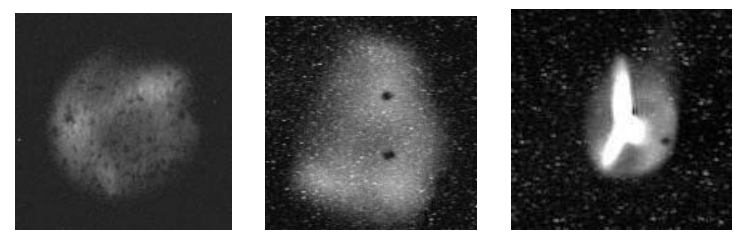

Figure 5: ${ }^{40} \mathrm{Ar}^{7+}$ images at $\mathrm{z}=10 \mathrm{~m}, 13 \mathrm{~m}$, and $16 \mathrm{~m}$, respectively. Beam intensity is cut by about half with a round aperture at $\mathrm{z}=7 \mathrm{~m}$ and the beam still appears round at $\mathrm{z}=10 \mathrm{~m}$, but redevelops its signature characteristics downstream.
Without careful preparation, a slit cut is equally futile, as the projected image of narrow slits is highly non-linear (Figure 6). This figure also demonstrates why $2 \mathrm{~d}$ emittance and position scans, while useful, cannot give a full description of beam characteristics.
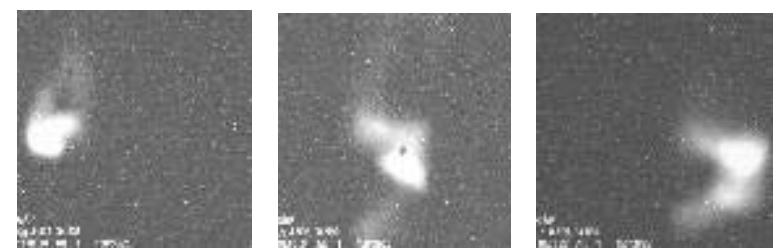

Figure 6: Scan left to right of the beam just in front of the $2 \mathrm{~d}$ emittance scanner with a horizontal slit gap of $2 \mathrm{~mm}$. The width of the beam on a viewer $50 \mathrm{~cm}$ downstream is about $1 \mathrm{~cm}$. A large $\mathrm{y}-\mathrm{x}$ correlation is shown. A $2 \mathrm{~d}$ emittance scan assumes the beam in this situation is a vertical line.

\section{SIMULATIONS}

Beam simulation presents major difficulties in the case where the object of the optical system is complicated. All codes operate under certain assumptions. Completely and accurately representing the atomic physical properties of ions and electrons and then tracking those particles through extraction and acceleration is a daunting task. However, we believe the following assumptions will allow modeling of ECRIS beams in a way that is both possible to do and accurate enough to make adequate predictions. Our present working assumptions are as follows:

- The "meniscus" is not suitable object for a simulated optical system.

- The extracted ions have a trajectory that is dominated by the magnetic fields through which they pass. They are not randomized by collisions.

- The object is deep. It is not an emission "disc" but rather an emission "cylinder."

- The extracted beam is hollow, with little intensity onaxis with low divergence.

Matrix-type codes allow for relatively easy optimization of a system of optical elements but can do that optimization only with relatively simple initial conditions on the starting beam. Full $3 \mathrm{~d}$ codes can track particles through complicated known fields but have long running times and have no provision for fitting the values of optical elements for a desired final condition. Given these limitations, we are using both types of codes for better understanding and as a guide to achieving better real world results.

\section{KOBRA-INP Simulations}

KOBRA3-INP uses the exact electric and magnetic fields, only influenced by the discreteness error, and without any symmetry restrictions. Space charge is taken into account by an iterative method. Inside the plasma chamber, charge neutrality is assumed. Ions are traced 
through the system using exact fields. An electron density distribution is generated analytically using Self's plasma model [6]. The full spectrum of all charge states and masses is treated simultaneously to get a correct spacecharge distribution.

The problem still remains to find the correct starting conditions for all ions. The best set of starting conditions achieved up to now has been found taking the full plasma chamber into consideration. Only ions created at a certain level of magnetic flux density can be extracted, due to the mirror field before extraction. The region where ions are launched has the form of a slightly deformed ellipsoid.

Projections of the $(\mathrm{x}, \mathrm{y})$ and $\left(\mathrm{x}^{\prime}, \mathrm{y}^{\prime}\right)$ phase spaces are shown in Figure 7 and show a large $2^{\text {nd }}$ order aberration exists on the extracted ECRIS beam.
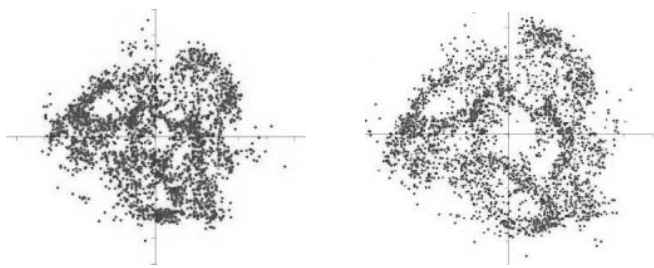

Figure 7: KOBRA3-INP simulation of ${ }^{40} \mathrm{Ar}^{3+}$ beam $20 \mathrm{~cm}$ from the extraction aperture of the CAPRICE ion source. The $(\mathrm{x}, \mathrm{y})$ space is shown at left $(4 \mathrm{~cm}$ beam diameter). At right, a plot in $\left(x^{\prime}, y^{\prime}\right)$ space shows the signature sextupole aberration with maximum divergence of about $90 \mathrm{mrad}$.

\section{TRANSPORT and GICOSY Simulations}

In these simulations, the object is an uncorrelated $8 \mathrm{~mm}$ diameter ${ }^{40} \mathrm{Ar}^{7+}$ beam of $240 \mathrm{pi}^{*} \mathrm{~mm} * \mathrm{mrad}$. A $2^{\text {nd }}$ order disturbance is generated by immediately passing the beam through an "ECRIS sextupole element. The rest of the line follows as shown in Figure 1, with the inclusion of a sextupole just in front of the analysis magnet, which will be physically installed in 2007. The purpose this addition is to fully correct $2^{\text {nd }}$ order effects induced by the sextupole within the ion source. An electrostatic doubletoctupole-doublet lens system was installed in late 2005 with this final goal in mind [7].

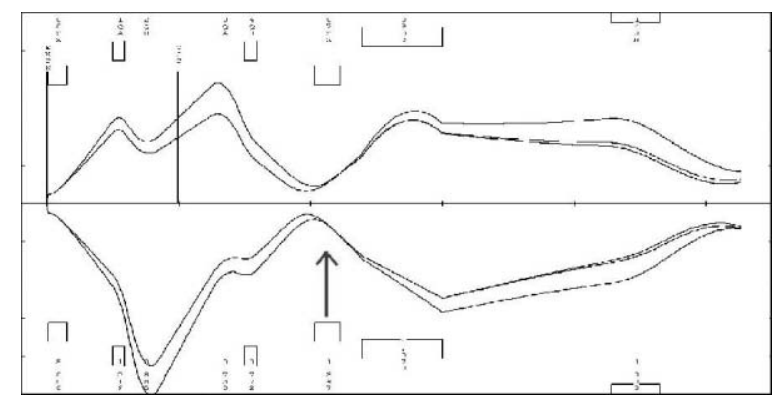

Figure 8: TRANSPORT envelopes of the ARTEMIS-A beam line to $\mathrm{z}=5 \mathrm{~m}$ showing nearly complete correction of the initial sextupole disturbance by using a corrector sextupole (indicated).
Figure 8 shows the TRANSPORT envelopes in $1^{\text {st }}$ order, $2^{\text {nd }}$ order, and $2^{\text {nd }}$ order corrected with the added beam line sextupole. The fitting is not easy and the stochastic fit option in the PSI Transport Framework was particularly useful in finding a good solution.

Moving the fitted values from TRANSPORT into GICOSY gives the same results and allows plotting phase-space information (Figure 9).

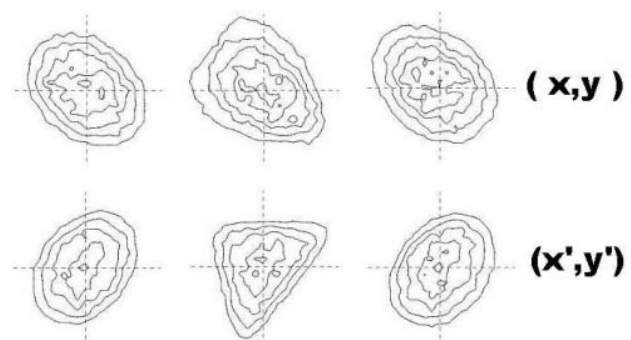

Figure 9: The upper plots show the beam in $(\mathrm{x}, \mathrm{y})$ space, the lower plots show $\left(x^{\prime}, y^{\prime}\right)$ space. Left to right are shown calculation results in $1^{\text {st }}$ order, $2^{\text {nd }}$ order, and $2^{\text {nd }}$ order with use of the corrector sextupole.

These simulations also show that $3^{\text {rd }}$ order effects can be important and are mitigated by use of octupole correction. In practice at NSCL, use of the octupole results in a $5-10 \%$ increase of beam intensity extracted from the K500 cyclotron.

\section{CONCLUSIONS}

Beams from ECR ion sources exhibit a complicated structure in $4 \mathrm{~d}$ space and cannot be modeled correctly unless this is taken into account. Viewer plates allow this structure to be observed more directly than with $\mathrm{x}, \mathrm{y}$ scanners, but future understanding must also come from $4 \mathrm{~d}$ emittance measurements. Possibilities exist for correction of $2^{\text {nd }}$ order effects and should be explored.

\section{ACKNOWLEDGEMENTS}

The authors wish to thank M. Doleans, G. Machicoane, F. Marti, M. Steiner, X.Y. Wu at NSCL and K. Tinschert and J. Mäder at GSI for their contributions.

\section{REFERENCES}

[1] KOBRA3-INP http://spaedtke@INP-DME.com

[2] U. Rohrer, "PSI Graphic Transport Framework", http://people.web.psi.ch/rohrer_u/trans.htm

[3] http://www-linux.gsi.de/ weick/gicosy/

[4] F. Marti, P. Miller, D. Poe, M. Steiner, J. Stetson, and X.Y. Wu, in Cyclotrons and their Applications 2001, AIP Conf. Proc. 600 (2001) p. 64.

[5] J. Yurkon, private communication, NSCL, 2006.

[6] S.A. Self, Exact Solution of the Collisionless PlasmaSheath Equation, The Physics of Fluids, 1963.

[7] J. Stetson, G. Machicoane, P. Miller, M. Steiner, P. Zavodszky, in Proc. of LINAC'06, 2007, p. 352. 